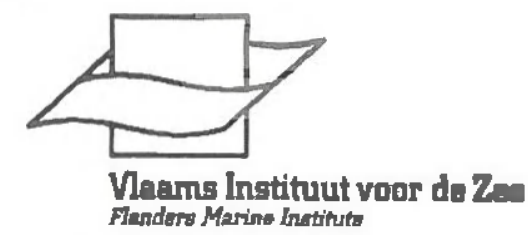

\title{
On the computation of the barotropic mode of a free-surface world ocean model
}

\author{
Eric Deleersnijder, Jean-Michel Campin \\ 23755 \\ Institut d'Astronomie et de Géophysique G. Lemaître (Unité ASTR), Université Catholique de Loưain, 2 Chemin du Cyclotron, \\ B-1348 Louvain-la-Neuve, Belgium
}

Received: 14 March 1994/Revised: 24 October 1994/Accepted: 8 November 1994

\begin{abstract}
The free-surface formulation of the equations of our world ocean model is brielly described. The barotropic mode equations are solved according to the split-explicit method, using different time steps for the external and internal modes. Because the numerical digorithm is implemented on the B-grid, a spurious. free-surface, two-grid interval mode may develop. This mode must be filtered out. The properties of two filters are theoretically investigated and their actual performance is tested in a series of numerical experiments. It is seen that one of these filters may severely perturb the local mass conservation, rendering it impossible to enforce the impermeability of the surface or the bottom of the ocean. The dynamics of the external mode is also examined, by studying the depth-integrated momentum equations. The depth-integral of the pressure force due to the slope of the ocean surface is approximately balanced by the depth-integral of the force ensuing from the horizontal variations of the density. The depth-integral of the Coriolis force is an order of magnitude smaller, except in the Southern Ocean. Two variational principles are resorted to for computing the fictitious ocean surface elevation corresponding to the approximate equilibrium between the dominant forces of the barotropic momentum equations.
\end{abstract}

\section{Introduction}

The large-scale ocean model devised by Bryan (1969) and Cox (1984) has now become a well-recognized standard. Other authors, such as Semtner $(1974,1986)$ or Delecluse et al. (1993) have built ocean models along similar guidelines. In the world ocean, these models were intended to perform integrations of several thousands of years, thus requiring that the time step be as large as possible. Since the time stepping selected is chiefly explicit, it is necessary to filter out the processes associated with the highest

Correspondence to: E. Deleersnijder propagation speeds which could have placed too stringent a limitation on the time increment. Therefore, the rigid-lid approximation is made to exclude the external inertiagravity wayes, the phase speed of which is of order $200 \mathrm{~m} \mathrm{~s}^{-1}$. As a result, the transport, i.e. the depth-integral of the horizontal velocity, is divergenceless and may be derived from a stream function. The latter is computed from a Poisson equation obtained by taking the curl of the depth-integral of the horizontal momentum equation. This approach, though widely used for about two decades, is no longer considered optimal.

Firstly, the solution procedure of the Poisson equation for the stream function may present several problems. Killworth and Smith (1984) pointed to possible instabilities in the iterative solution procedure of the stream function equation. In the latter equation, a factor proportional to the inverse of the ocean depth was also shown to be detrimental to the numerical method (Dukowicz et al., 1993). Finally, the nature of the boundary condition applied at the coastline of islands implies the evaluation of non-local integrals, leading to data transfers that can seriously slow down modern distributed-memory computers (Dukowicz et al., 1993). It is also worth mentioning that Neuman pressure problems are standard in NavierStokes solvers and are certainly much to be preferred to the stream function formulation.

Secondly, the ocean surface elevation is not directly accessible, which could imply difficulties for the assimilation of altimeter data, a subject to which, in recent years, an increasing research effort is being devoted (e.g. Holland and Malanotte-Rizzoli, 1989; Mellor and Ezer, 1991; Verron,1992; Verron et al., 1992; Schröter et al., 1993).

To find a remedy to the drawbacks of the stream function approach, two methods were suggested.

In the first one, the rigid-lid approximation was kept, but the divergence, instead of the curl, of the depth-integrated momentum equation was taken, leading to a Poisson equation for the surface pressure which is better conditioned from a numerical and computational point of view. Indeed, steep bottom slopes are more easily taken into account and the boundary conditions for the pressure 
equation, which are of the Neuman type, lead to local computations only (Gresho and Sani, 1987; Deleersnijder and Campin, 1993; Dukowicz et al., 1993; Pinardi et al., 1995).

The second alternative method no longer relied on the rigid-lid approximation. In other words, the ocean surface was considered free and, hence, became one of the prognostic variables of the model. This could allow the inclusion of tides and the study of the inverted barometer eflect. On the other hand, to overcome the severe limitation of the time step due to the presence of fast-propagating external Poincaré waves (Beckers and Deleersnijder, 1993), the split-explicit method (Gadd, 1978; Madala, 1981) may be called on, as was done in our free-surface ocean model (Berger et al., 1993; Deleersnijder and Campin, 1993), as well as in other models (Blumberg and Mellor, 1987; Beckers, 1991; Killworth et al., 1991). As was argued by Killworth et al. (1991), performing several hundred iterations for solving a Poisson equation to evaluate the stream function or the surface pressure may take the same amount of computer time as that needed to update the barotropic mode variables with a time step much smaller than that of the baroclinic mode.

The present article addresses several aspects of the computation of the barotropic mode in our free-surface world ocean model. First, the equations of the barotropic mode are recalled. Then, since the B-grid is used, it is shown that a checkerboard mode in the surface elevation may develop. This mode is a mere artefact which must be filtered out. The properties of two filters are theoretically investigated. Their impact on the amplitude of the spurious mode and on the rertical velocity is examined in a series of numerical experiments. Finally, the dominant terms of the barotropic momentum equation are identified

\section{Barotropic mode}

Our ocean general circulation model (Berger et al., 1993; Deleersnijder and Campin, 1993) was recently set up and will be described in detail in a forthcoming article. Here, it suffices to say that the equations of the model are similar to those of the most classical models (Bryan, 1969; Semtner, 1974, 1986). Pacanowski and Philander (1981) formulae are used 10 parameterize the vertical turbulent diffusion. The only significant difference lies in the treatment of the barotropic mode, in which the ocean surface is considered free.

In the following discussion, the term barotropic mode or external mode is used to denote the motion associated with the depth-averaged horizontal velocity. The variables of this mode are depth-independent and comprise the ocean surface elevation $\xi$ and the transport $\boldsymbol{U}$. The baroclinic or internal mode is concerned with the deviation of the horizontal velocity relative to its depth-average, as well as the temperature and the salinity. This way of separating the modes is not equivalent, though very close, to the classical splitting performed in a linearized model with flat bathymetry (Gill, 1982). This point has been touched upon by Killworth et al. (1991), but we believe that, because the difference between the two definitions is generally very small, there is no cause for concern.

According to the hydrostatic equilibrium, the pressure $p$ is given by

$p=p_{a}+g \int_{z}^{\xi} \rho \mathrm{d} z$

where $p_{a}, g, \rho$, and $z$ denote the atmospheric pressure at sea level, the gravitational acceleration, the water density, and the vertical coordinate (pointing upwards), respective$1 \mathrm{y} ; \xi$ is positive when the free surface is above the reference ocean level, where $z=0$. Since $\xi$ is much smaller than the sea depth $h$, and since $\rho$ is always very close to its reference value $\rho_{0}$, Eq. (1) may be approximated by

$p=p_{a}+g \rho_{0} \xi+g \int_{z}^{0} \rho \mathrm{d} z$.

The atmospheric pressure may be expressed as $p_{a}=\bar{p}_{a}+\hat{p}_{a}$, where $\bar{p}_{a}$ and $\hat{p}_{a}$ denote the average of $p_{a}$ over $\Omega$, the ocean surface, and the deviation relative to this mean, respectively. It is convenient to define the reference ocean level so that the average of $\xi$ over $\Omega$ be zero. For world ocean simulations phenomena having timescales no larger than a few days may be filtered out. Thus, the inverted barometer effect may be assumed valid (Ponte, 1993), implying that $\hat{p}_{a}$ is balanced by the "inverted barometer part of $\xi^{\prime \prime}$, which we denote $\xi^{i b}$. As a consequence, $\xi$ may be written as

$\xi=\xi^{i b}+\eta$,

with

$\hat{p}_{a}+g \rho_{0} \xi^{i b}=0$,

implying that the average of $\eta$ over $\Omega$, the ocean surface, is zero.

The horizontal pressure gradient force reads

$$
-\frac{1}{\rho_{0}} \nabla_{p}=-g \nabla \eta-\frac{g}{\rho_{0}} \int_{z}^{0} \nabla \rho \mathrm{d} z .
$$

The gradient operator is defined as

$\nabla=\sec \theta e_{x} \frac{\partial}{\partial x}+e_{y} \frac{\partial}{\partial y}$,

with $(x, y)=a(\lambda, \theta)$, where $a, \lambda$ and $\theta$ represent the Earth radius, longitude and latitude, respectively; unit vectors $\boldsymbol{e}_{x}$ and $\boldsymbol{e}_{y}$ are tangent to the Earth surface and point eastwards and northwards, respectively.

In the remainder of this article, we will only deal with the "non-barometric part of $\xi ", \eta$, which, for simplicity, will be termed ocean surface elevation.

Mass conservation requires

$\frac{\partial \eta}{\partial t}=-\nabla \cdot \boldsymbol{U}$,

where $t$ is time. The divergence operator $\nabla \cdot$ represents

$\nabla \cdot=\sec \theta\left[\frac{\partial}{\partial x}\left(\boldsymbol{e}_{\boldsymbol{x}} \cdot+\frac{\partial}{\partial y}\left(\cos \theta \boldsymbol{e}_{y} \cdot\right.\right.\right.$ 
The depth-integral of the horizontal momentum equations reads

$\frac{\partial \boldsymbol{U} T}{\partial t}=\boldsymbol{F}_{\eta}+\boldsymbol{F}_{p}+\boldsymbol{F}_{c}+\boldsymbol{D}(\boldsymbol{U})+\boldsymbol{A}$.

In the momentum budget above

$\boldsymbol{F}_{\eta}=-g h \nabla \eta$

is the force due to the slope of the ocean surface, while

$\boldsymbol{F}_{\rho}=-\int_{-h}^{0}\left(\frac{g}{\rho_{0}} \int_{z}^{0} \nabla \rho \mathrm{d} z\right) \mathrm{d} z$

and

$\boldsymbol{F}_{c}=-f \boldsymbol{e}_{z} \times \boldsymbol{U}$

denote the force produced by the horizontal inhomogeneities of the density and the Coriolis force, respectively. The operator $\boldsymbol{D}$ is of a diffusive nature and involves horizontal derivatives only so that $\boldsymbol{D}$ may be a horizonal Laplacian or bi-Laplacian. Finally, $\boldsymbol{A}$ encompasses the remaining terms ensuing from the depth-integration of the horizontal momentum equation, i.e. advection and bottom, surface stress terms. Some details about the numerical scheme are given in Appendix A.

\section{Filtering the checkerboard mode}

The B-grid allows the existence and the possible growth of a spurious two-grid interval free-surface mode of the following type

$\eta_{i, j}^{c}=E^{c}(t)(-1)^{i+j}$,

where $E^{c}(t)$ represents the amplitude of the spurious "checkerboard" mode $\eta^{c} ; i$ and $j$ are integer indices traditionally associated with the horizontal discretization - in the $x$ and $y$-direction, respectively. It is straightforward to show that the checkerboard mode is associated with zero pressure gradient in the numerical algorithm, which is the reason that it can develop and persist.

Clearly, a mode like $\eta^{c}$ corresponds to a mere numerical artefact which must be filtered out.

As pointed out by Kilworth et al. (1991), the gridsplitting problem has been addressed several times in the atmospheric literature. Nonetheless, solutions that are valid in the atmosphere are unlikely to be relevant to ocean models, primarily because of the presence of irregular lateral boundaries, i.e. the coasts.

Killworth et al. (1991) suggested adding to the righthand side of the discretized counterpart of Eq. (7) a term of the form

$K_{i, j}=\frac{A_{H}^{\eta}}{S_{i, j}}\left(A_{i, j}-\delta B_{i, j}\right)$,

where $A_{H}^{\eta}$ is an appropriate diffusivity; $S_{i, j}$ denotes the area of the $(i, j)$ grid box; $\delta$ is equal to 1 in Killworth et al. (1991) but, by setting $\delta=0$, a classical Laplacian-type filter is obtained; $A_{i, j}$ and $B_{i, j}$ are defined in Eqs. (A3) and
(A4) in Killworth et al. (1991), i.e.,

$$
A_{i, j}=\left(\eta_{i-1, j}+\eta_{i+1, j}-2 \eta_{i, j}\right)+\left(\eta_{i, j-1}+\eta_{i, j+1}-2 \eta_{i, j}\right),
$$

$$
\begin{aligned}
B_{i, j}= & \frac{1}{2}\left[\left(\eta_{i-1, j-1}+\eta_{i+1, j+1}-2 \eta_{i, j}\right)\right. \\
& \left.+\left(\eta_{i-1, j+1}+\eta_{i+1, j-1}-2 \eta_{i j}\right)\right] .
\end{aligned}
$$

The additional terms represent a smoother reaching its maximum efficiency for a mode of the form $\eta_{i, j}^{c}$. This is easily seen.

If $S_{i, j}$ is constant, $A_{i, j}$ and $B_{i, j}$ may be interpreted as two alternative discretizations of the Laplacian of $\eta, A_{i, j}$ using derivatives along the coordinate axes and $B_{i, j}$ resorting to diagonal expressions. Subtracting $B_{i, j}$ from $A_{i, j}$ thus amounts to evaluate the difference between two discretized Laplacians.

To understand the properties of the filter suggested by Killworth et al. (1991), it is convenient to examine the behaviour of the following discrete equation

$\frac{\eta_{i, j}^{n+1}-\eta_{i, j}^{n}}{\Delta t}=\alpha\left(A_{i, j}^{n}-\delta B_{i, j}^{n}\right), \quad n=0,1,2, \ldots$,

where index " $n$ " refers to the instant $n \Delta t, \Delta t$ being the time step. We consider Fourier components of the variable $\eta$, i.e.,

$\eta_{i, j}^{n}=\operatorname{Re}\left\{E^{n} \exp [I(i k+j l)]\right\}$,

with $I=(-1)^{1 / 2}$ and $0 \leq k, l \leq \pi, k$ and $l$ denoting dimensionless wave numbers. In the " $i$ " direction for example. the two-grid interval mode is associated with $k=\pi$, while the mode with an infinite length scale corresponds to $k=0$. The chekerboard mode is that for which $k=\pi=l$. Substituting Eq. (18) into Eqs. (15)-(17), we obtain the expression of the gain $G$ of the filter for the mode identified by $(k, l)$

$$
\begin{aligned}
G= & \frac{E^{n+1}}{E^{n}}=1-2 \alpha \Delta t[2-\cos k-\cos l \\
& -\delta(1-\cos k \cos l)] .
\end{aligned}
$$

To prevent time oscillations from arising $G$ must remain positive. It is readily seen that this condition is satisfied if and only if $8 \alpha \Delta t \leq 1$.

As stated above, if we put $\delta=0$ we have a classical Laplacian-type filter, the maximum damping of which is obtained for $k=\pi=l$, i.e., for the checkerboard mode. This filter leaves the infinitely long numerical waves unaffected. The $\delta=1$ filter shares these properties with the Laplacian filter. However, the $\delta=1$ smoother is clearly preferable since it induces less damping on all the modes, except the checkerboard mode and the $k=0=l$ mode. Since

$2-\cos k-\cos l>1-\cos k \cos l$ for $(k, l) \neq(\pi, \pi)$

and $(0,0)$,

we indeed have

$G(\delta=1)>G(\delta=0)$ for $(k, l) \neq(\pi, \pi)$ and $(0,0)$.

The properties of the $\delta=1$ and $\delta=0$ filters are illustrated in Fig. 1. 

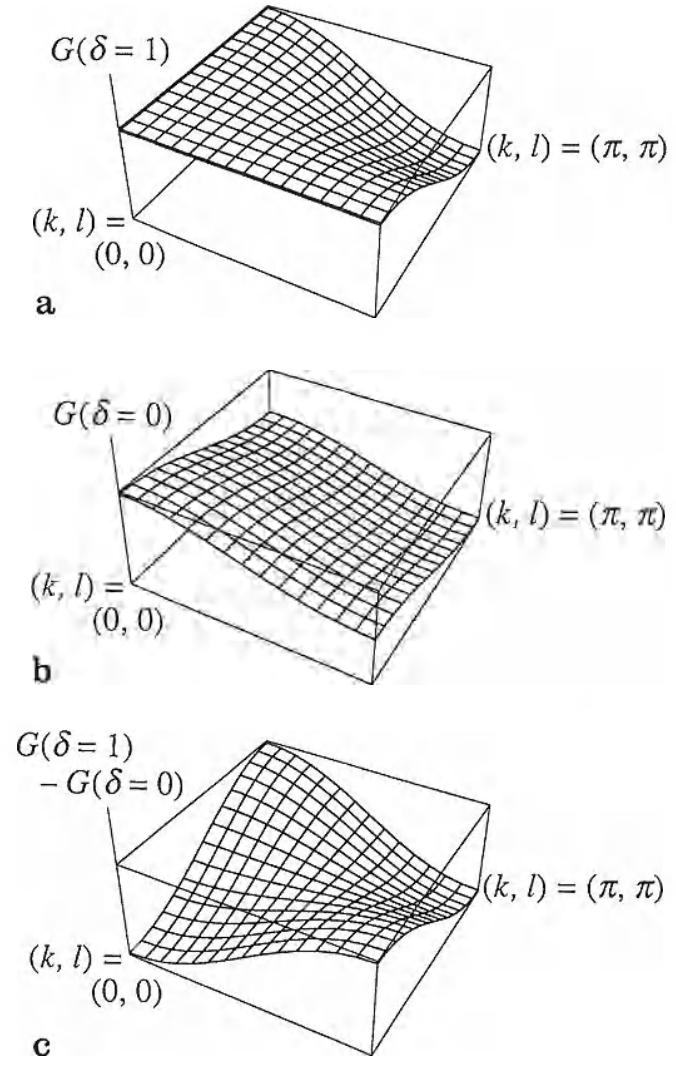

Fig. 1. Perspective view of the gain $G$ of the $\delta=0$ filter a and b of the $\delta=1$ filter. c The difference $G(\delta=1)-G(\delta=0)$ clearly illustrates that the $\delta=1$ smoother involves less damping

So far the filters have been examined in a two-dimensional unbounded domain. To deal with the irregular boundaries limiting oceanic computational domains modifications are to be introduced. They must ensure overall mass conservation, i.e. the modified filter must not include sinks or sources of $\eta$ in the vicinity of the coastlines. Killworth et al. (1991) have suggested modifications that, at first sight, might seem to be somewhat intricate. Here we propose a strategy that is perhaps more straightforward, relying on a conservative formulation.

It is assumed that two land masks are implemented in order to obtain a vectorisable computer programme. One, $m^{\eta}$, is associated with the elevation points, while the other, $m^{u, v}$, is defined at the same location as the horizontal components of the velocity (Fig. 2a). The mask $m^{\eta}$ is defined as follows

$m_{i, j}^{\eta}=1$ if wet grid box,

$m_{i, j}^{\eta}=0$ if dry grid box.

Hence, according to the specifications of the B-grid (Fig. 2a),

$m_{i-1 / 2, j-1 / 2}^{u, v}=m_{i, j}^{\eta} m_{i-1, j}^{\eta} m_{i-1, j-1}^{\eta} m_{i, j-1}^{\eta}$.

It must be stressed that $m^{\eta}$ and $m^{u, t}$ are likely to be used in most B-grid ocean models. Those masks are thus not specifically designed for the purpose of filtering a spurious free-surface mode.

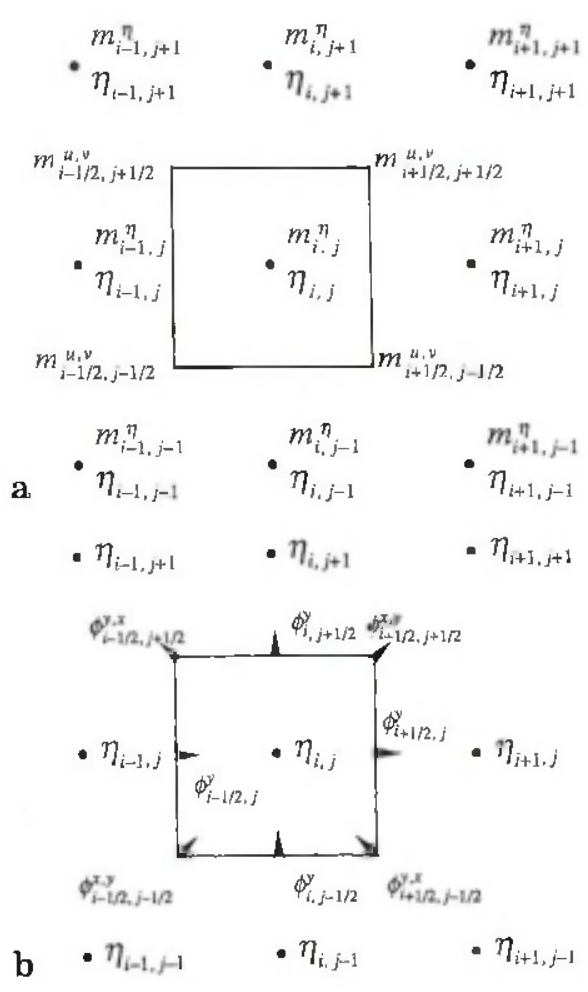

Fig. 2. a Location on the B-grid of land masks $m^{n}$ and $m^{u, v}$ and $\mathbf{b}$ of the fluxes $\phi^{x}, \phi^{y}, \phi^{x, y}$ and $\phi^{y, x}$

Upon defining appropriate fluxes,

$\phi_{i-1 / 2, j}^{x}=-m_{i, j}^{\eta} m_{i-1, j}^{\eta}\left(\eta_{i, j}-\eta_{i-1, j}\right)$,

$\phi_{i, j-1,2}^{\eta}=-m_{i, j}^{\eta} m_{l, j-1}^{\eta}\left(\eta_{i, j}-\eta_{i, j-1}\right)$,

$\phi_{i-1 / 2, j-1 / 2}^{x, y}=-m_{i-1 / 2, j-1 / 2}^{u, v}\left(\eta_{i, j}-\eta_{i-1, j-1}\right)$,

$\phi_{i+1 / 2, j-1 / 2}^{5, x}=-m_{i+1 / 2, j-1 / 2}^{u, v}\left(\eta_{i, j}-\eta_{i+1, j-1}\right)$,

one may cast $A_{i, j}$ and $B_{i, j}$ into a conservative form

$A_{i, j}=-\phi_{i+1 / 2, j}^{x}+\phi_{i-1 / 2, j}^{x}-\phi_{i, j+1 / 2}^{y}+\phi_{i, j-1 / 2}^{y}$,

$B_{i, j}=\frac{1}{2}\left(-\phi_{i+1 / 2, j+1 / 2}^{x_{1}, p}+\phi_{i-1 / 2, j-1 / 2}^{x, y}\right.$

$-\phi_{i-1 / 2, j+1 / 2}^{y, x}+\phi_{i+1 / 2, j-1 / 2}^{y, z}$.

Away from any lateral solid boundary, Eqs. (24)-(29) are equivalent to the operators suggested in Killworth et al. (1991).

As shown in Fig. 2b, the fluxes are defined on the boundaries of the grid boxes. Moreover, with the help of the masks, every flux that is defined at a dry point is set to zero, whatever the fictitious value of $\eta$ on the land. It is then readily understood that the expressions detailed above guarantee overall mass conservation in the external mode.

It is difficult to determine whether our operator still has smoothing properties in the vicinity of a lateral boundary. The only analysis that can be easily carried out pertains to the pure checkerboard mode $\eta_{i, j}^{c}$. This mode does not 
contribute to the diagonal fluxes $\phi^{x, y}$ and $\phi^{y, x}$. Hence, $B\left(\eta^{c}\right)$ is zero. We then have

$$
\begin{aligned}
K\left(\eta_{i, j}^{c}\right)= & -2 \frac{A_{H}^{\eta}}{S_{i, j}}\left(m_{i-1, j}^{\eta} m_{i, j}^{\eta}+m_{i, j}^{\eta} m_{i+1, j}^{\eta}\right. \\
& \left.+m_{i, j-1}^{\eta} m_{i, j}^{\eta}+m_{i, j}^{\eta} m_{i, j+1}^{\eta}\right) \eta_{i, j}^{c},
\end{aligned}
$$

which proves that the operator $K$ always tends to filter out the checkerboard mode, irrespective of the presence of a lateral boundary.

Theoretical analysis of the filters is clearly not sufficient. Numerical expetiments must be carried out so as to evaluate the amplitude of the checkerboard mode, the impact of the smoothers and their possible secondary effects.

\section{Numerical experiments}

The smoothers described herein have been used in our world ocean model, running on a spherical $5^{\prime \prime} \times 5^{\circ}$ grid with realistic topography (Deleersnijder and Campin, 1993) (Fig. 3). In the version of the model discussed herein, the Arctic Ocean was not included, but a truly global and finer grid (Deleersnijder et al., 1993) is presently operational. There were nine levels in $z$-coordinate in the vertical direction. The model was forced with the annual mean of the wind stress taken from the data set of Hellerman and Rosenstein (1983). The integrations of the model were conducted in a robust-diagnostic mode. In other words, the temperature and salinity fields were forced to remain close to the annual means of the Levitus (1982) climatology. To do so, restoring terms with a timescale of 2 years were introduced in the equations governing the evolution of potential temperature and salinity. No surface fluxes of temperature and salinity were explicitly imposed. but the restoring timescale was set to 15 days in the grid box adjacent to the ocean surface.

Numerical experiments were performed for various values of $A_{H}^{\eta}$, with $\delta=0$ and $\delta=1$. All results analyzed below correspond to steady-state solutions of the governing equations.

To assess the impact of the filters, it is necessary to evaluate the local amplitude of the checkerboard mode. Accordingly, the sea surface elevation is expressed as

$\eta_{i, j}=\eta_{i, j}^{c_{i} l}+\eta_{i, j}^{\prime}$,

where $\eta_{i, j}^{c, l}$ is the local checkerboard contribution, which is obtained from

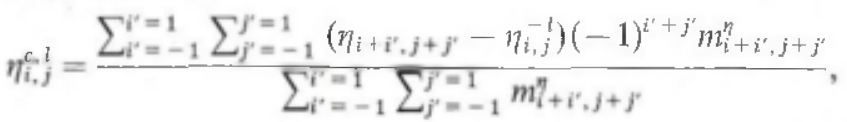

with

$\eta_{i, j}^{-l}=\frac{\sum_{\vec{l}=-1}^{j^{\prime}=1} \sum_{j^{\prime}=-1}^{j^{\prime}=1} \eta_{i+i^{\prime}, j+j^{\prime}} m_{i+i^{\prime}, j+j^{\prime}}^{\eta^{\prime}}}{\sum_{i^{\prime}=-1}^{j^{\prime}=1} \sum_{j^{\prime}=1}^{i^{\prime}=1} m_{i+i^{\prime}, j+j^{\prime}}^{\eta}}$.

One may think of many ways to locally evaluate the amplitude of the checkerboard mode. Formulation (32) seems, however, quite natural, since it is based on the projection of the actual sea surface elevation field onto a local, pure checkerboard field $(-1)^{i^{+}+j^{\prime}}$. Furthermore, definition (32) involves the nine points that are concerned by the $\delta=1$ filter and is able to cope with the presence of solid boundaries.

In the present study, a global measure of a variable " $a$ " is obtained by taking its root mean square over its computational domain, which we denote $|a|_{\text {RMS }}$.

Table 1 shows that the global measure of $\eta^{c .1}$ decreases as $A_{H}^{\eta}$ increases, as expected. With $\delta=1$, the amplitude of the checkerboard mode is slightly higher than with $\delta=0$. This is apparently not in agreement with the gain of the filter being independent of $\delta$ for the checkerboard mode.

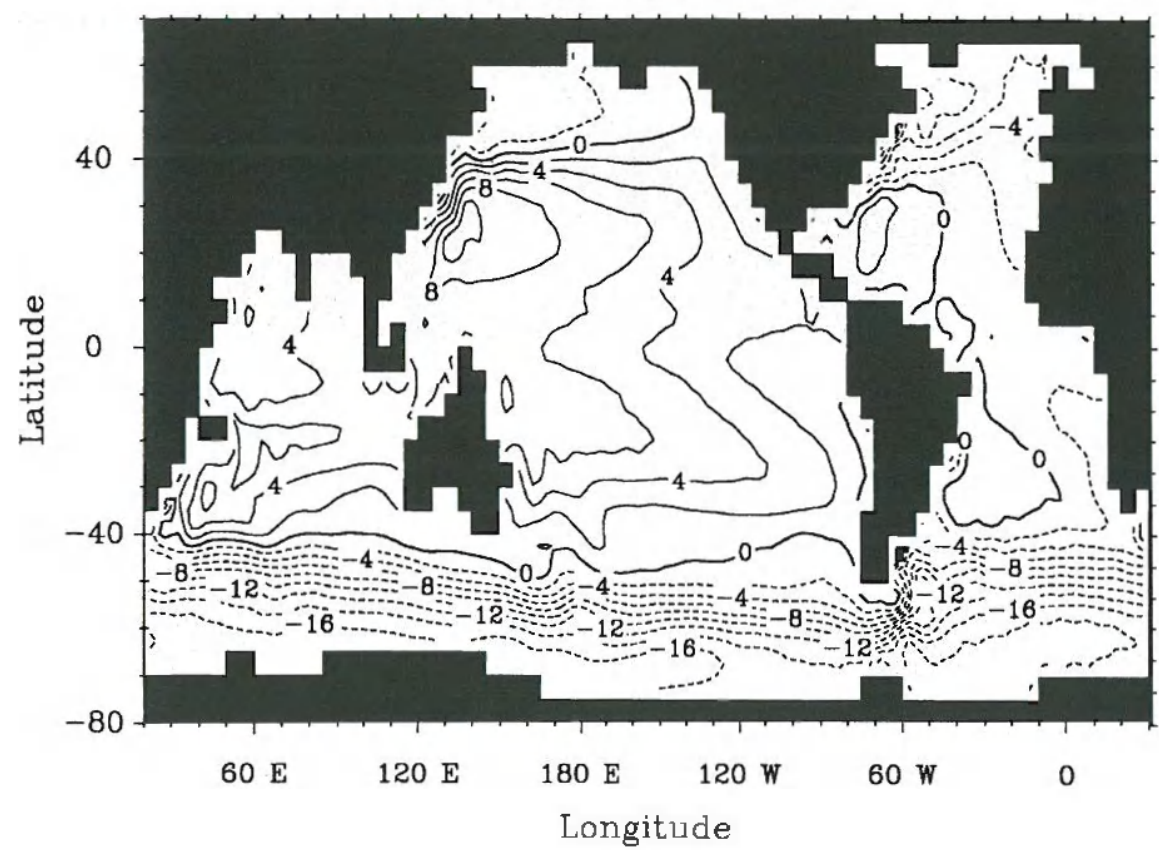

Fig. 3. Steady state ocean surface elevation computed with $A_{H}^{\eta}=2 \times 10^{5} \mathrm{~m}^{2} \mathrm{~s}^{-1}$ and $\delta=1$ (in $0.1 \mathrm{~m}$ ) 
Table 1. Global evaluation of the amplitude of the checkerboard mode and the spurious vertical velocity at the ocean bottom

\begin{tabular}{llllll}
\hline $\begin{array}{l}A_{G}^{n} \\
\left(10^{5} \mathrm{~m}^{2} \mathrm{~s}^{-1}\right)\end{array}$ & $\delta$ & $\begin{array}{l}\left|\eta \eta^{c, l}\right|_{R M S} \\
\left(10^{-2} \mathrm{~m}\right)\end{array}$ & $\frac{\left|\eta^{c, 1}\right|_{R M S}}{|\eta|_{R M S}}$ & $\begin{array}{l}\left|w_{b}\right|_{R M S} \\
\left(10^{-9} \mathrm{~m} \mathrm{~s}^{-1}\right)\end{array}$ & $\frac{\left|w_{b}\right|_{R M S}}{|w|_{R M S}}$ \\
\hline 0.0 & & 2.74 & 0.0427 & 0.0359 & 0.00295 \\
0.5 & 0 & 2.19 & 0.0341 & 0.0215 & 0.0178 \\
0.5 & 1 & 2.24 & 0.0347 & 0.0156 & 0.0129 \\
1.0 & 0 & 2.05 & 0.0319 & 0.410 & 0.0341 \\
1.0 & 1 & 2.13 & 0.0329 & 0.290 & 0.0239 \\
2.0 & 0 & 1.85 & 0.0289 & 0.767 & 0.0647 \\
2.0 & 1 & 1.97 & 0.0305 & 0.531 & 0.0440 \\
4.0 & 0 & 1.61 & 0.0253 & 1.41 & 0.121 \\
4.0 & 1 & 1.77 & 0.0275 & 0.943 & 0.0785 \\
10.0 & 0 & 1.29 & 0.0205 & 3.12 & 0.275 \\
10.0 & 1 & 1.48 & 0.0230 & 1.93 & 0.161 \\
\hline
\end{tabular}

Several causes may explain this minor discrepancy between theory and numerical experiments. First, the above definition of the local amplitude of the checkerboard mode has some questionable aspects. Second, the Fourier analysis that led to Eq. (19) of the gain of the filter pertains to the simplified Eq. (17) considered in an unbounded domain. This is an idealized approach, which may not be straightforwardly applied to a realistic case. The correct way of reasoning is probably as follows: since the $\delta=1$ filter involves in general less damping, it is not surprising that the small-scale components of $\eta$ have a somewhat higher magnitude in the experiments where $\delta=1$ than in those with $\delta=0$.

In Table 1, it may be seen that, even when no filter is used $\left(A_{H}^{n}=0\right)$, the amplitude of the checkerboard mode is very small compared with the total sea surface elevation. Indeed, the ratio $\left|\eta^{c, l}\right|_{R M S}|\eta|_{\text {RMS }}$ does not exceed $\approx 4.3 \%$. For the simulations presented here, the filter is probably not necessary. However, numerical experiments recently carried out with the new version of our model, covering the Arctic and having a finer resolution, i.e. $3 \times 3$, are much more prone to the development of high-amplitude checkerboard mode. The filter will thus not be remored from our model. Instead, it will be considered an optional tool to be activated when needed.

In the numerical experiments discussed here, $A_{H}^{\eta}$ ranged from 0 to $10^{6} \mathrm{~m}^{2} \mathrm{~s}^{-1}$, while the horizontal viscosity and diffusivity were equal to $200 \times 10^{3} \mathrm{~m}^{2} \mathrm{~s}^{-1}$ and $10^{3} \mathrm{~m}^{2} \mathrm{~s}^{-1}$, respectively.

The vertical velocity $w$ is obtained by integrating over $z$ the local mass conservation equation

$\nabla \cdot\left(\frac{U}{h+\eta}+\hat{u}\right)+\frac{\partial w}{\partial z}=0$

where $\hat{\boldsymbol{u}}$ is the deviation of the horizontal velocity relative to its depth-average. The vertical velocity derived from Eq. (34) is such that the three-dimensional velocity $\boldsymbol{v}=\boldsymbol{U} /(h+\eta)+\hat{\boldsymbol{u}}+\boldsymbol{w} \boldsymbol{e}_{z}$ is divergenceless. If, in addition, $\eta$ and $\boldsymbol{U}$ satisfy the integrated continuity equation, Eq. (7), then it is readily seen that $v$ is able to meet the impermeability conditions of the bottom and the surface of the ocean. However, when a filter is active, the barotropic continuity equation, Eq. (7), is perturbed. Thus, if Eq. (34) is integrated downwards from the ocean surface, the bottom impermeability condition will no longer be verified. Conversely, if $w$ is computed by integrating the local mass conservation equation from the ocean bottom, it is the surface impermeability condition that will not be met.

The ocean bottom was not assumed to be flat in our simulations. However, because of the particular location of the variables on the B-grid, the bottom vertical velocity on a vertical where scalar quantities are computed must be zero. Note also that, unlike Cox (1984) or Semtner (1986), we did not separately evaluate the vertical velocity to be used in the momentum budget. Instead, we computed this vertical velocity by a simple four-point average, ensuring that the vertical velocity field of the scalar verticals agrees with that of the momentum verticals.

In our model, the vertical velocity is obtained from a downward space marching beginning with zero vertical velocity at $z=0$. Because this starting point is slightly erroneous, but extremely easy to implement in our numerical algorithm in which the displacements of the free surface are actually taken into account only in Eq. (7), the vertical velocity at the bottom, $w_{b}$, cannot be zero, as it should be (Table 1). When the filter is disabled $\left(A_{H}^{\eta}=0\right)$, $w_{b}$ is truly negligible. When a filter is active the higher $A_{H}^{u}$ is, the larger the order of magnitude of $w_{b}$ is (Table 1). In other words, the stronger the filter the larger the perturbation to the bottom impermeability condition. For a given value of $A_{H}^{\eta}$, the $\delta=0$ filter induces "leaks" through the bottom that are significantly higher than with the $\delta=1$ filter. This may be explained as follows. Since we are examining steady-state solutions obtained with a filter acting on the ocean surface elevation, we have

$(\nabla \cdot U)_{i, j}=K_{i, j}$.

At the grid point where $w_{b}$ is evaluated, the bathymetry gradient is locally considered to be zero. Hence, integrating Eq. (34) downwards from the surface reference level, neglecting the gradient of $\eta$, using Eq. (35), we obtain

$\left(w_{b}\right)_{i, j}=K_{i, j}$.

Considering a Fourier component of $\eta$ as in Eq. (18), $\left(w_{b}\right)_{i, j}$ may be written as

$\left(w_{b}\right)_{i, j}=\frac{-2 W_{b}}{\sqrt{S_{i, j}}} \operatorname{Re}\left\{E^{n} \exp [I(i k+j l)]\right\}$,

with

$W_{b}=\frac{A_{I I}^{\eta}}{\sqrt{S_{i, j}}}[2-\cos k-\cos l-\delta(1-\cos k \cos l)]$.

This strongly suggests that $\left|w_{b}\right|_{R M S}$ could approximately be a linear function of $A_{H}^{\eta}$, i.e.

$\left|w_{b}\right|_{R M S} \approx r(\delta) A_{H}^{\eta}$.

By virtue of inequality Eq. (20), $W_{b}(\delta=1) \leq W_{b}(\delta=0)$, implying that the slope $r$ should be higher when $\delta=0$

$r(\delta=1) \leq r(\delta=0)$.

A simple inspection of Table 1 shows that relations (39) and $(40)$ are rather well corroborated by the model results. 


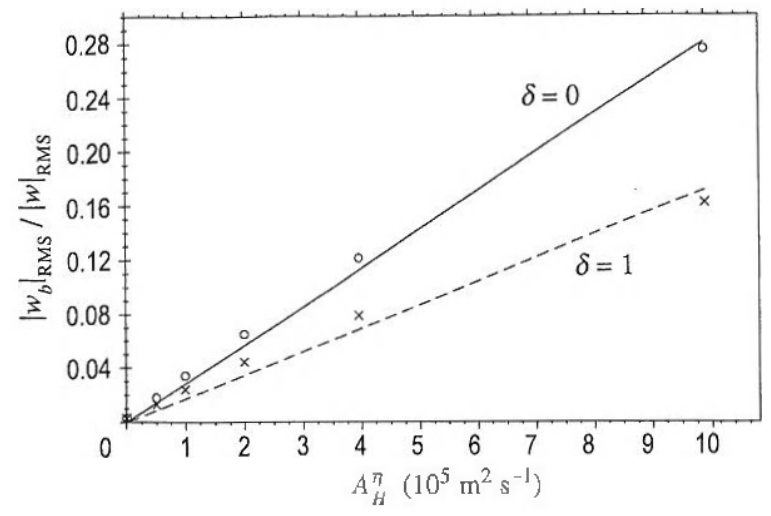

Fig. 4. Plot of the ratio $\left|w_{b}\right|_{\text {RMS }} /|w|_{\text {RMS }}$ as a function of $A_{H}^{n}$ for the $\delta=1$ filter $(x)$ and the $\delta=0$ filter (O). The lines are ohtained from least square analysis of the model results, which leads to $\left|w_{b}\right|_{R M S}\left|w^{\prime}\right|_{R M S}=0.017 \times 10^{-5} A_{H}^{\eta} \quad$ and $\quad\left|w_{b}\right|_{R M S} /|w|_{R M S}=$ $0.028 \times 10^{-5} A_{Y Y}^{n}$ for $\delta=0$, respectively

The order of magnitude of $w_{b}$ does not really matter as such. It is more important to determine whether or not the spurious vertical velocity at the bottom is comparable to the vertical velocily in the interior of the ocean. Hence, $\left|w_{b}\right|_{R M S} / \mid w_{i R M S}$ is more relevant than $\left|w_{b}\right|_{R M S}$ (Table 1). Away from the bottom, the vertical velocity is more influenced by the divergence of the deviation of the horizontal velocity than by its depth-average. Thus, we may expect $|w|_{R M S}$ to be fairly constant throughout the serjes of numerical experiments we performed. This is indeed the case. Therefore, the ratio $\left|w_{b}\right|_{R M S}|w|_{R M S}$ must have the same properties as $\left|w_{h}\right|_{R M S}$, as is clearly confirmed by Fig. 4.

Obviously, the magnitude of $w_{b}$ must remain very small in order to avoid damaging the evolution of scalar quantities. Determining the highest acceptable value of $x_{b}$ is a priori difficult. One would suggest that the ratio $\left|w_{b}\right|_{R M S}|w|_{R M S}$ should not exceed a few percent, implying that, for the present series of numerical experiments, $A_{H}^{n}$ should not be larger than approximately $200 \mathrm{~m}^{2} \mathrm{~s}^{-1}$. For the latter value of $A_{H}^{\eta}$ and with the $\delta=1$ filter, the elevation field predicted by the model is displayed in Fig. 3. For this set of parameters, the horizontal barotropic circulation (Fig. 5) and the meridional overturning motion (Fig. 6) are similar to those obtained by other coarse grid models in robust-diagnostic mode.

The filter suggested by Killworth et al. (1991) is superior to a classical Laplacian-type filter, not because of its impact on the checkerboard mode, but because it is less detrimental to other variables, in particular the vertical velocity. The primary reason is that the $\delta=1$ filter involves less damping, except for the checkerboard mode, as was shown above.

Overall the volume of the world ocean is conserved because the filter is implemented in a conservative way and because the corresponding fluxes are zero at the coastline. There are, however, local losses or gains of water through the botrom. This is the reason that we insisted that the vertical velocity at the bottom, $w_{b}$, should be as small as possible. We have also implemented a numerical trick for correcting temperature/salinity drifts that may be induced by spurious flow convergence or divergence in the bottom grid box. This corrective method is working well, as long as $w_{b}$ is sufficiently small. All this is a truly minor problem!

Finally, it must be pointed out that the ocean surface topography predicted by the model is in agreement, at least from a qualitative point of view, with that recently measured by the Topex/Poseidon altimeters (Fig. 7).

\section{Dominant force in the momentum budget}

To understand the mechanisms governing the motion of the ocean surface, it is instructive to examine the role of

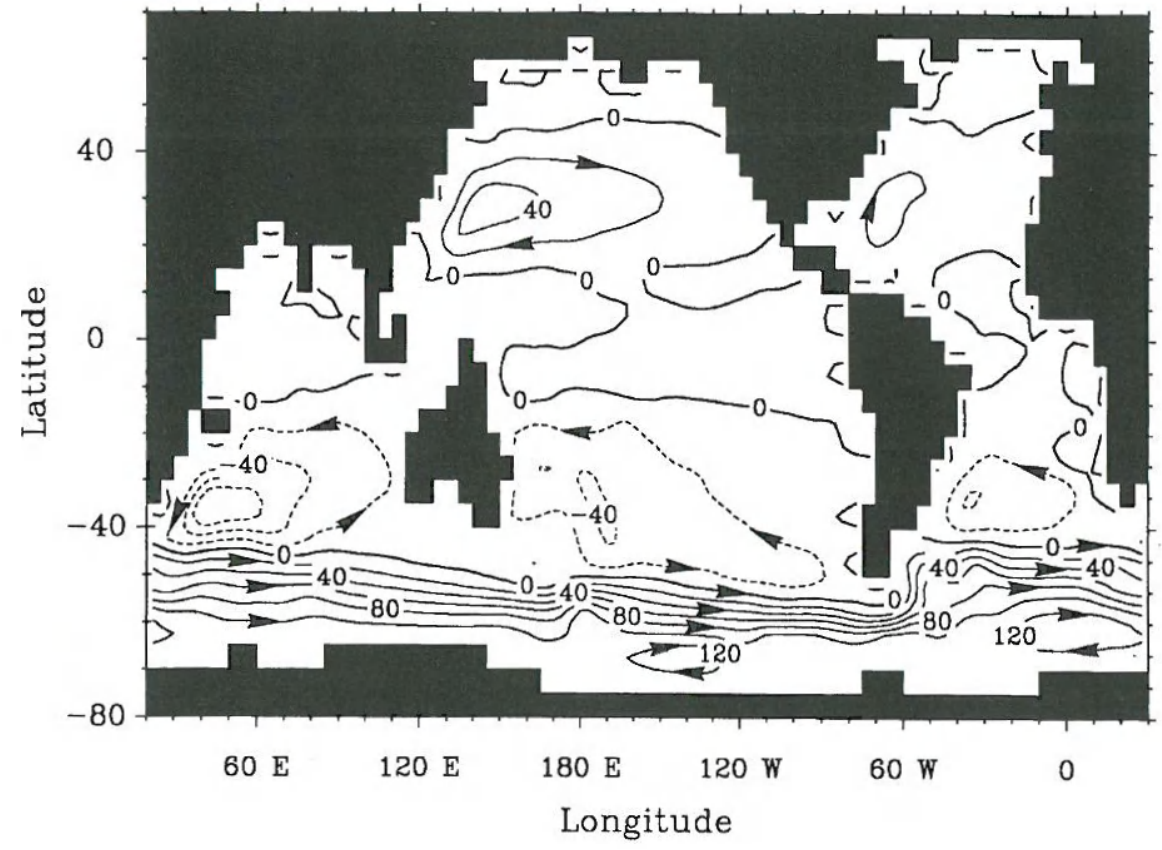

Fig. 5. Steady-state barotropic circulation computed with $A_{H}^{\eta}=2 \times 10^{5} \mathrm{~m}^{2} \mathrm{~s}^{-1}$ and $\delta=1$ (in Sverdrups) 


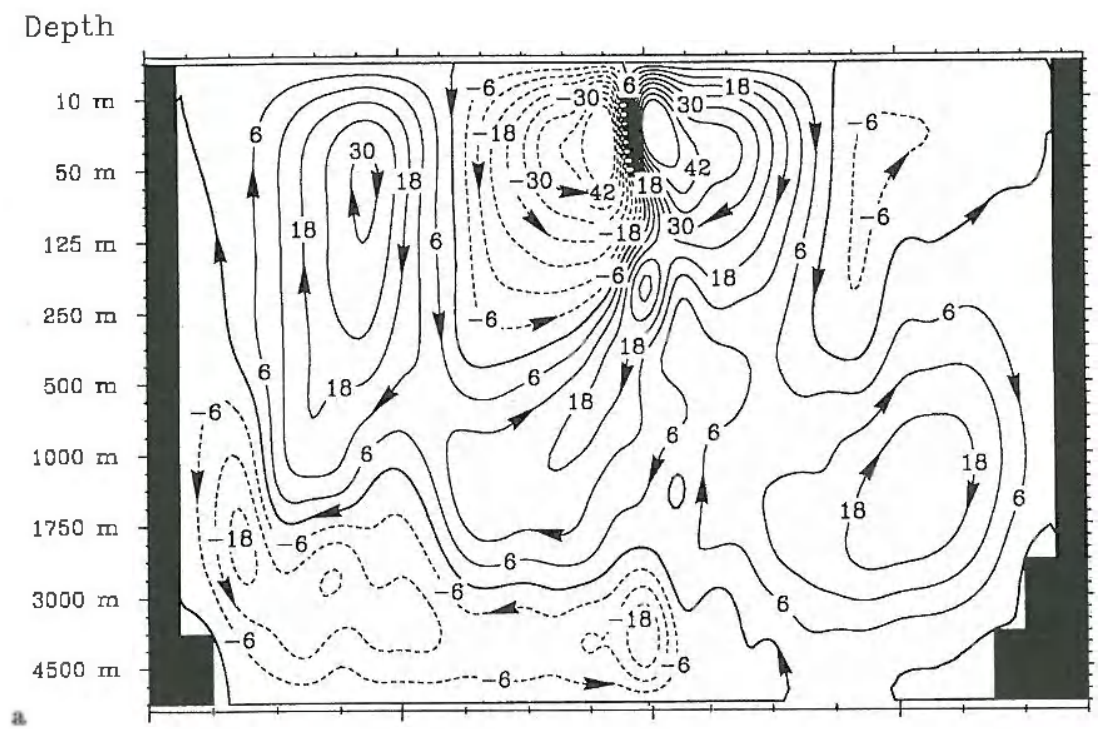

a

Depth

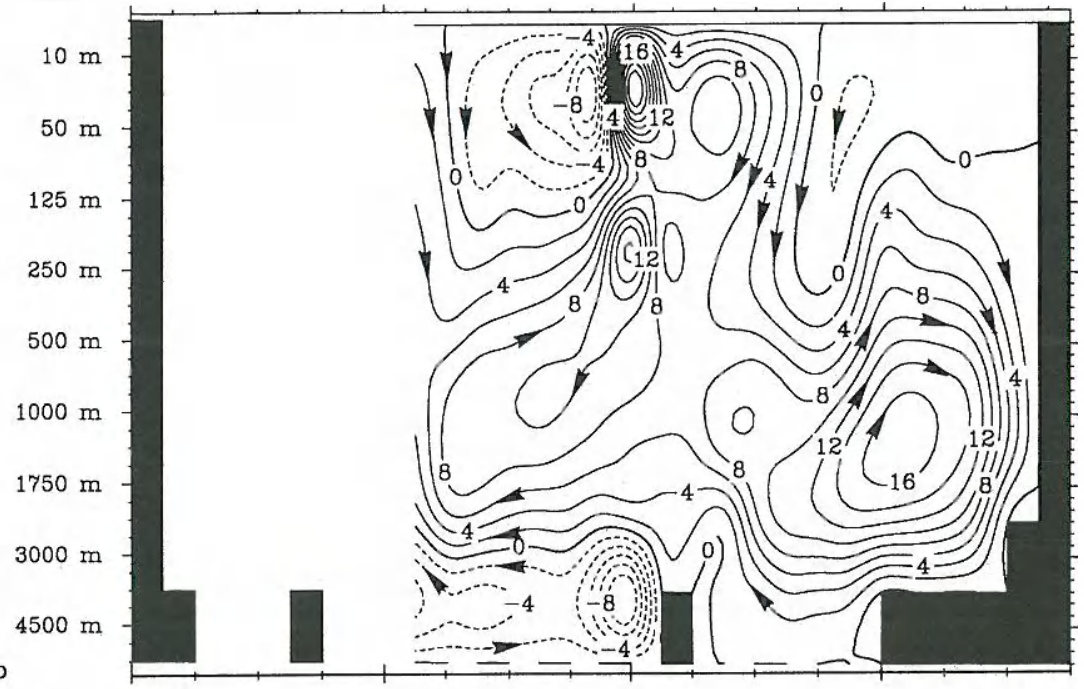

b

Depth

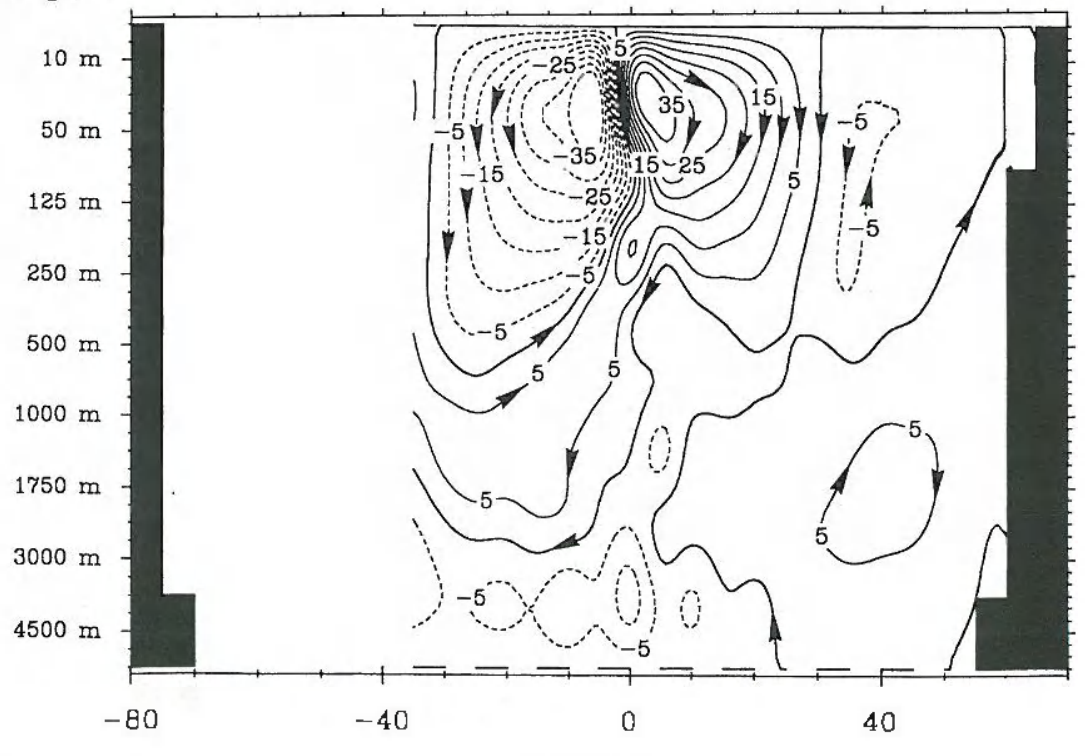

Fig. 6a-c. Steady-state meridional overturning circulation computed with $A_{H}^{n}=2 \times 10^{5} \mathrm{~m}^{2} \mathrm{~s}^{-1}$ and $\delta=1$ (in Sverdrups). a The global mean together with $\mathbf{b}$ the Atlantic and $\mathbf{c}$ the Indo-Pacific circulations are displayed c

Latitude 


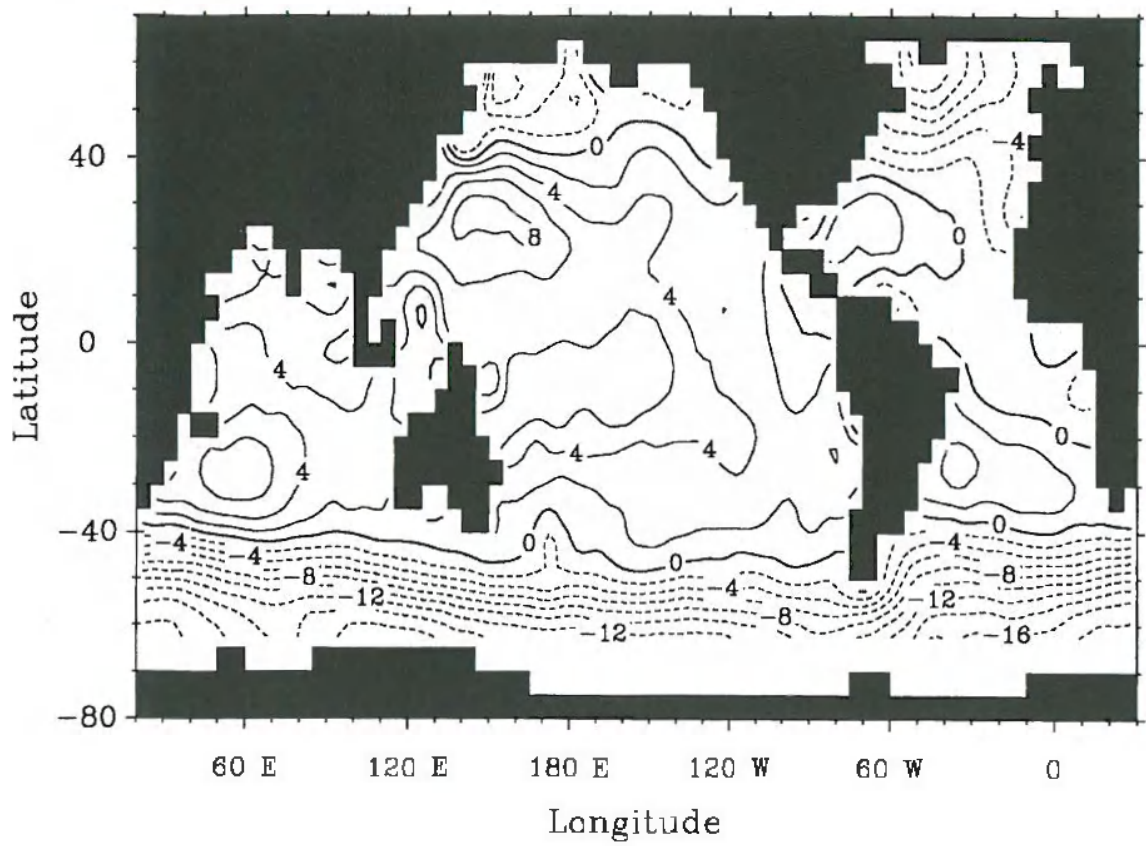

Fig. 7. Average of 1 year of Topex Poseideon data, i.e. from Cycle 2 (October 1992) until Cycle 38 (October 1993). The original data have been somewhat smoothed and interpolated onto our numerical grid. The ocean surface elevation is given in $0.1 \mathrm{~m}$

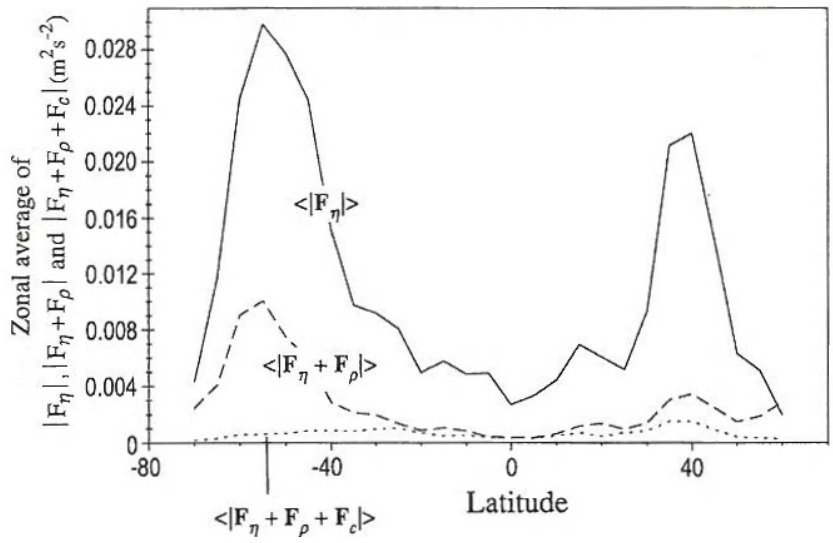

Fig. 8. Zonal average of $\left|\boldsymbol{F}_{\eta}\right|$ (solid curve), $\left|\boldsymbol{F}_{\eta}+\boldsymbol{F}_{\phi}\right|$ (dashed curve) and $\left|\boldsymbol{F}_{n}+\boldsymbol{F}_{p}+\boldsymbol{F}_{c}\right|$ (dotted curve)

the forces driving the transport $\boldsymbol{U}$. We investigate the dynamics of the external mode, in particular, our attention will be focused on the identification of the forces dominating the momentum equation, Eq. (9).

Forces $\boldsymbol{F}_{\eta}, \boldsymbol{F}_{\rho}$ and $\boldsymbol{F}_{c}$ are examined. According to their definitions, i.e. Eqs. (10)-(12), they represent depth-integrated forces which are due to the slope of the ocean surface, the horizontal variations of density and the Coriolis effect, respectively.

Figure 8 clearly shows that

$$
\left|\boldsymbol{F}_{\eta}+\boldsymbol{F}_{\rho}+\boldsymbol{F}_{c}\right| \ll\left|\boldsymbol{F}_{\eta}+\boldsymbol{F}_{\rho}\right| \ll\left|\boldsymbol{F}_{\eta}\right| .
$$

The forces $\boldsymbol{F}_{r_{1}}$ and $\boldsymbol{F}_{p}$ approximately balance each other, except in the region of the Antarctic Circumpolar Current where the Coriolis force is also significant. In other words, the barotropic mode is not dominated by the geostrophic equilibrium, but by the balance of two pressure forces. The imbalance of these forces is, in turn, approximately in geostrophic equilibrium

From the view point of the external mode, $\boldsymbol{F}_{\rho}$ appears as a forcing term, i.e. a force which does not directly depend on $\eta$ or $\boldsymbol{U}$. This force may be split as

$h\left(\boldsymbol{F}_{\rho} / h\right)=h\left(\boldsymbol{F}_{\rho} / h\right)^{\mathrm{d}}+h\left(\boldsymbol{F}_{\rho} / h\right)^{\mathrm{r}}$,

where the superscript $d$ refers to the divergent part of $F_{\rho} / h$ and $r$ identifies its rotational part. That $\boldsymbol{F}_{\eta}$ and $\boldsymbol{F}_{\rho}$ roughly balance each other actually means that

$g \nabla \eta \approx\left(\boldsymbol{F}_{\rho} / h\right)^{\mathrm{d}}$,

and that

$\left|\left(\boldsymbol{F}_{\rho t} / h\right)^{\mathrm{d}}\right| \geqslant\left|\left(\boldsymbol{F}_{\rho} / h\right)^{\mathrm{r}}\right|$.

Since $\left(\boldsymbol{F}_{\rho} / h\right)^{\mathrm{d}}$ has zero curl it is dynamically equivalent to the forcing term due to the atmospheric pressure. Consequently, $h\left(\boldsymbol{F}_{\rho /} / h\right)^{\mathrm{d}}$ may be written in terms of an equivalent atmospheric pressure force, i.e.

$h\left(\boldsymbol{F}_{\rho} / h\right)^{\mathrm{d}}=-\frac{h}{\rho_{0}} \nabla p_{a}^{e q}$.

Combining Eqs. (43) and (45) yields

$\hat{p}_{a}^{\text {eq }}+g \rho_{0} \eta \approx 0$.

The latter expression is formally equivalent to that defining the inverted barometer effect, i.e. Eq. (4). Therefore, the external mode dynamics is dominated by phenomena that are similar to those associated with the inverted barometer effect. The primary role of the external inertia-gravity waves is, therefore, to restore the equivalent inverted barometer equilibrium (Eq. (46)). In addition, this adjustment must be as quick as that prevailing in the proper inverted barometer effect, i.e. its timescale is of the order of a few days (Ponte, 1993). 
One may want to evaluate the fictitious elevation fields expressing that $\boldsymbol{F}_{\eta} \approx-\boldsymbol{F}_{\rho}$ and, more accurately, that $\boldsymbol{F}_{\eta} \approx-\boldsymbol{F}_{\rho}-\boldsymbol{F}_{6}$. As will be seen, this will produce another illustration of how well these forces balance each other.

It is worth recalling that the present analysis is based on model results only. Therefore, no claim is made that the "truth" has been found out. This is all the more important that the seasonal cycle is not taken into account, which might well reinforce the importance of the static forces.

It is suggested seeking the elevation field $\eta_{1}$, which is such that a global measure of $\left|\boldsymbol{F}_{\eta}+\boldsymbol{F}_{\rho}\right|$ is minimum, where $\boldsymbol{F}_{\eta}$ is then evaluated with $\eta_{1}$. Many global measures of $\left|\boldsymbol{F}_{n}+\boldsymbol{F}_{0}\right|$ may be considered, but we select $\left\|\boldsymbol{F}_{\eta}+\boldsymbol{F}_{\rho}\right\|_{R M S}$ because it allows elementary mathematical manipulations, as will be seen.

We thus require that $\eta_{1}$ be such that the functional

$J_{1}=\int_{\Omega}\left|-g h \nabla \eta_{1}+\boldsymbol{F}_{\rho}\right|^{2} \mathrm{~d} \Omega$

be minimum. The Euler-Lagrange equations of this variational principle are

$\nabla \cdot\left(g h^{2} \nabla \eta_{1}\right)=\nabla \cdot\left(h \boldsymbol{F}_{\rho}\right)$, in $\Omega$

$\boldsymbol{n} \cdot \nabla \eta_{1}=0$, on $\Gamma$

where $\Gamma$ is the boundary of $\Omega$ and $\boldsymbol{n}$ denotes the unit vector normal to $\Gamma$. The boundary condition, Eq. (49), derives from $F_{\rho}$ being considered zero on $\Gamma$. Equations (48)-(49) form an elliptic partial differential problem, the solution of which is close to the elevation field predicted by the model, with $A_{H}^{\eta}=200 \times 10^{3} \mathrm{~m}^{2} \mathrm{~s}^{-1}$ and $\delta=1$. We indeed have

$\frac{\left|\eta_{1}-\eta\right|_{R M S}}{|\eta|_{R M S}}=0.15$
If the transport is known, the minimum of another functional may be sought, i.e.

$J_{2}=\int_{\Omega}\left|-g h \nabla \eta_{2}+\boldsymbol{F}_{p}+\boldsymbol{F}_{c}\right|^{2} \mathrm{~d} \Omega$.

The Euler-Lagrange equations of this problem are similar to Eqs. (48)-(49), provided $\boldsymbol{F}_{\rho}$ be replaced by $\boldsymbol{F}_{\rho}+\boldsymbol{F}_{c}$. As expected, $\eta_{2}$, the elevation field achieving the minimum of functional (51), is closer to $\eta$ than $\eta_{1}$. Comparison of $\eta_{2}$ and $\eta$ yields $\left|\eta_{2}-\eta\right|_{R M S}|\eta|_{R M S}=0.019$, so that

$\frac{\left|\eta_{2}-\eta\right|_{R M S}}{\left|\eta_{1}-\eta\right|_{R M S}}=0.13$

Strictly speaking, $\eta_{1}$ and $\eta_{2}$, as computed from the appropriate Euler-Lagrange equations, are defined up to an additive constant, for the partial differential problems only involve derivatives of $\eta_{1}$ and $\eta_{2}$. There is thus no absolute reference for these elevation fields. Therefore, $\eta_{m}$ $(m=1,2)$ may be written as $\eta_{m}=\mu_{m}(x, y)+c_{m}$, where $c_{m}$ is a constant. It seems reasonable to require that $\eta_{m}$ be as close as possible to $\eta$. It follows that $c_{m}$ must be such that $\eta$ and $\eta_{m}$ have the same average over $\Omega$, i.e.

$c_{m}=\Omega^{-1} \int_{\Omega}\left(\eta-\mu_{m}\right) \mathrm{d} \Omega, \quad m=1,2$.

Figures 9 and 10 displays global maps of $\eta_{1}-\eta$ and $\eta_{2}-\eta$, respectively.

It must be pointed out that $\eta_{1}$ and $\eta_{2}$ were actually not computed from the discretized counterparts of the EulerLagrange equations set out above. Instead, we minimized the discrete equivalents to the functionals $J_{1}$ and $J_{2}$. The latter strategy allows us to consider discrete expressions of the forces that are similar to those used in the circulation model, thus leading to more appropriate fields of $\eta_{1}$ and $\eta_{2}$. For example, $\left|\eta_{1}-\eta\right|_{\text {RMS }}$ is about twice larger when $\eta_{1}$ is derived from the discretized version of Eqs. (48)-(49).

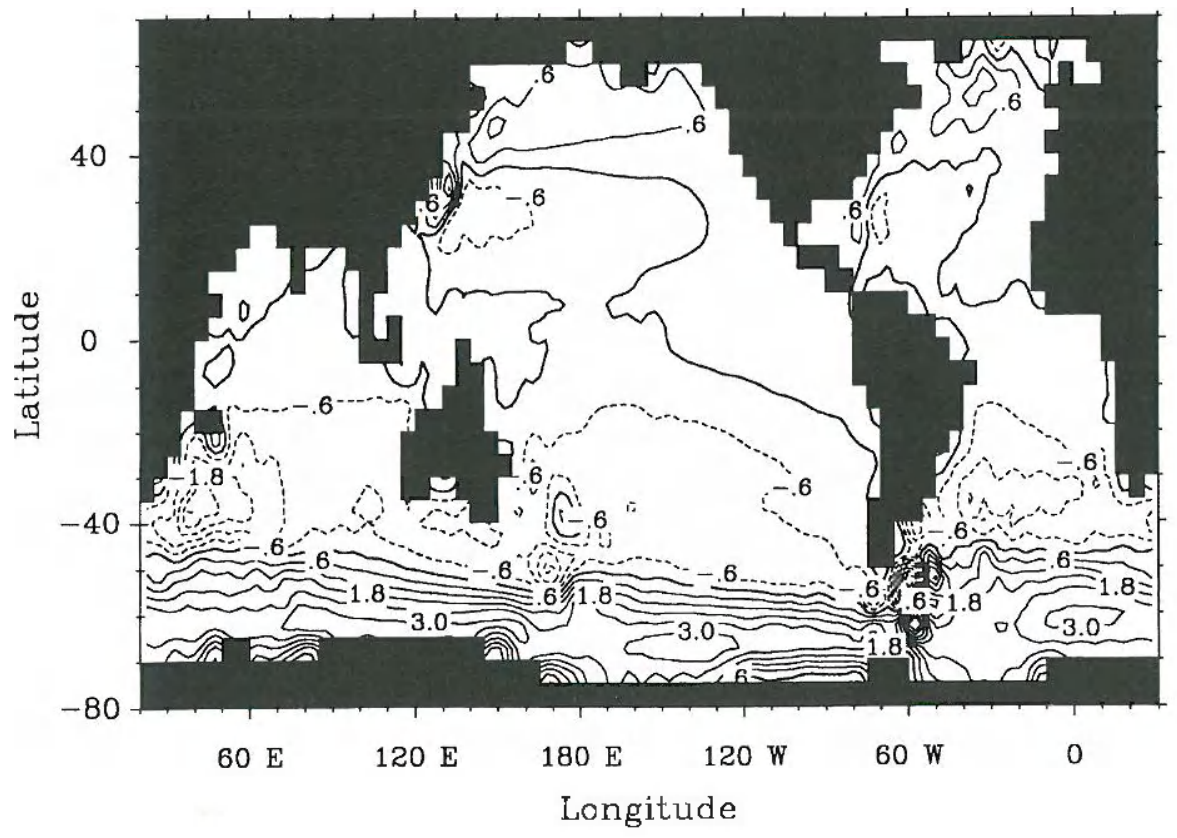

Fig. 9. Map of $\eta_{1}-\eta$ (in $\left.0.1 \mathrm{~m}\right)$ 


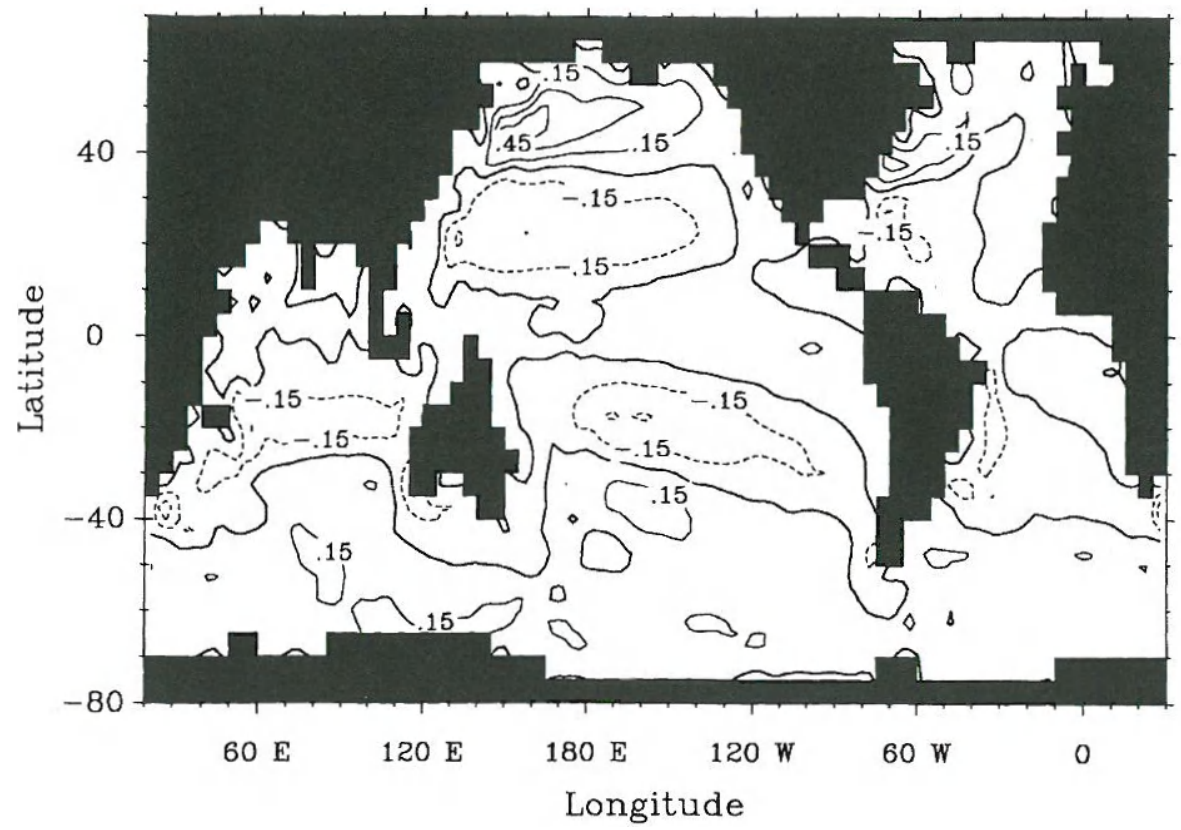

Fig. 10. Map of $\eta_{2}-\eta$ (in $0.1 \mathrm{~m}$ )

It is instructive to compare our variational technique with the classical method for evaluating the dynamic height from hydrographic data. The latter rests on the hypothesis that there exists a level of no geostrophic motion. Accordingly, it is assumed that, at a given distance $d$ to the ocean surface, the horizontal pressure gradient is zero. This leads to a third approximation to the ocean surface elevation, which we denote $\eta_{3}$. A simple manipulation of Eq. (5) leads to

$\eta_{3}=-\int_{-d}^{0} \frac{\rho}{\rho_{0}} \mathrm{~d} z+c_{3}$

where constant $c_{3}$ is determined so that $\eta_{3}$ be as close as possible to $\eta$. implying that $\eta_{3}$ and $\eta$ must have the same average. Obviously, in the regions where $d$ is larger than the ocean depth, the classical method cannot provide any estimation of the surface elevation. Furthermore, there is no irrefutable way of prescribing $d$. Accordingly, we tested the sensitivity of $\eta_{3}$ to various values of $d$, which was successively taken to be the depth of the grid points where

Table 2. Relative difference between $\eta_{3}$ and $\eta$ obtained with various values of the level of zero horizontal pressure gradient, $d$. The fraction of the ocesn surface where $d<h$ is denoted $s$

\begin{tabular}{rll}
\hline $\mathrm{d}(\mathrm{m})$ & $s$ & $\frac{\left|\eta_{3}-\eta\right|_{\text {RMS }}}{|\eta|_{\text {RMS }}}$ \\
\hline 22.1 & & 0.96 \\
68.4 & 1.00 & 0.87 \\
159.0 & 1.00 & 0.75 \\
339.0 & 0.98 & 0.60 \\
704.0 & 0.97 & 0.43 \\
1400.0 & 0.96 & 0.24 \\
2470.0 & 0.94 & 0.10 \\
3760.0 & 0.91 & 0.11 \\
5170.0 & 0.83 & 0.27 \\
\hline
\end{tabular}

the vertical velocity is computed (Table 2). It appeared that $\eta_{3}$ was never closer to $\eta$ than $\eta_{2}$. The hest approximation was obtained for $d=2470 \mathrm{~m}$ (Fig. 11). In this case, $\eta_{3}$ was somewhat better than $\eta_{1}$, but $\eta_{3}$ could not be evaluated over $9 \%$ of the ocean surface, where $d$ was deeper than the ocean bottom.

\section{Discussion and conclusion}

No thorough analysis of the time variability of the ocean surface elevation has been achieved in the present study. For example, no time spectrum of $\eta$ was produced and examined. This is obviously coherent with steady-state results being sought. One may, bowever, believe that the ocean surface elevation should vary according to at least two timescales, which should be several orders of magnitude apart from each other. The shorter timescale, i.e. no more than a few days, should concern external inertiagravity waves involved in the adjustment towards the equivalent inverted barometer equilibrium. The longer timescale should be that of the variations of the equivalent atmospheric pressure, i.e. the timescale of the density variations.

Assuming that the governing equations of the barotropic mode are sufficiently linear, the barotropic motions may be split into two categories, those associated with the atmospheric pressure forcing and those induced by the other forcings, of which the dominant is due to the horizontal density gradient. Enforcing the inverted barometer equilibrium amounts to abandoning representing the fast varying part of $\xi^{\text {ib }}$. This implies that, in our model, the short timescale variations of $\eta$ are irrelevant, i.e. they cannot be compared with in situ measurements. Consequently, it is only the slow part of $\eta$ that is, hopefully. physically meaningful. On a longer timescale the rigid lid approximation is likely to hold true. Thus, one may 


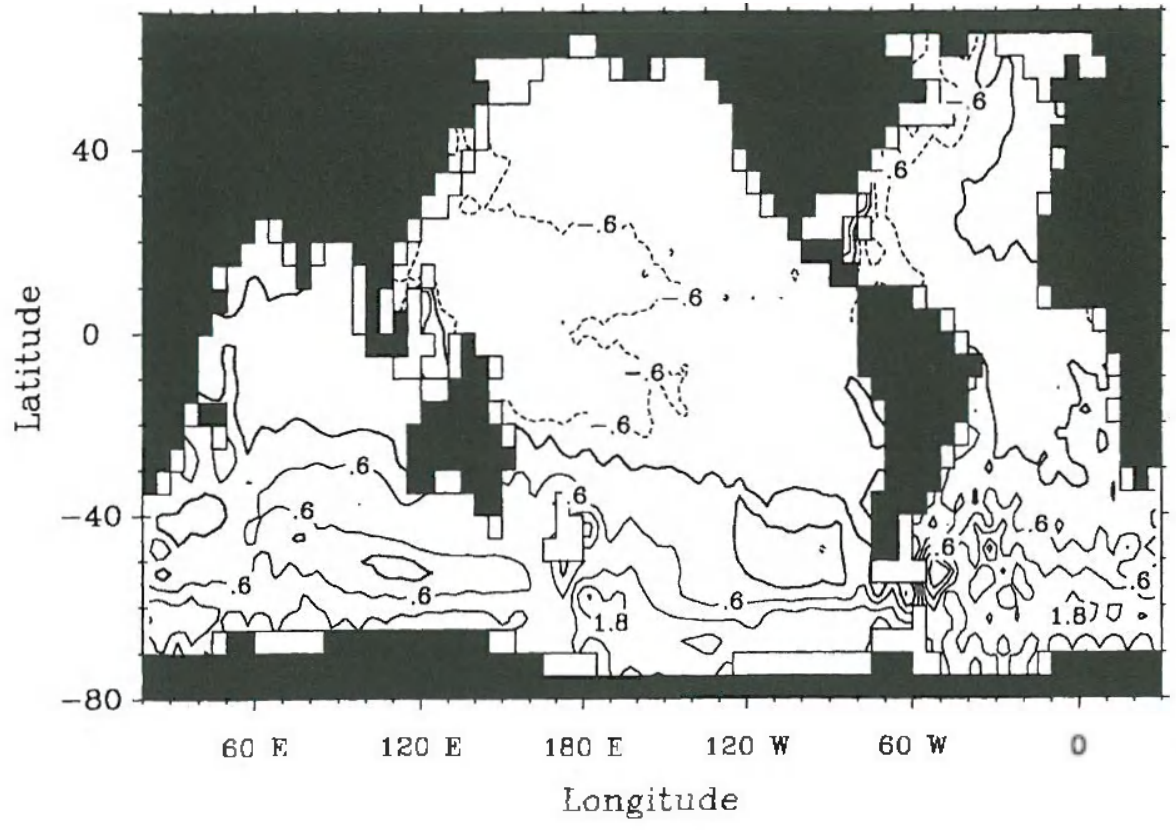

Fig. 11. Map of $\eta_{3}-\eta$ for $d=2470 \mathrm{~m}$ (in $0.1 \mathrm{~m})$ wonder whether it is really desirable to consider the ocean surface as free. In other words, one might think that the best modelling choice would be to compute the surface pressure in the framework of the rigid-lid approximation. A complete answer is yet to be provided.

One might consider the free-surface formulation as a mere numerical technique for computing the surface pressure which actually does not add any extra physical information to the model. The split-explicit approach indeed bears strong similarity with the artificial compressibility method for solving the Poisson equation for the surface pressure (e.g. Peyret and Taylor, 1983). However, such a method is unlikely to be optimal as regards the CPU time needed, since there exist numerous Poisson solvers that are much more efficient. From this standpoint, what is necessary is a thorough comparison of the computational performance of various numerical approaches. In the simulations discussed herein, about $10 \%$ of the CPU time was devoted to the computation of the external mode.

On the other hand, it must be kept in mind that a free-surface model has more potential than a rigid lid one. Indeed, it could allow the study of short timescale phenomena such as tides and inverted barometer adjustment. In addition, a free-surface model could straightforwardly be coupled with models of the shelf seas surrounding the deep oceans. Those shallow water areas represent less than $1 \%$ of the volume of sea water but are now believed to play a very significant role in the global biogeochemical processes. Hence, in the near future, it could be found desirable to set up a modelling system covering both the deep and shallow seas, in which case implementing free-surface models would most probably be found desirable.

To conclude, it may be thought that a free-surface model is more appropriate if short timescale phenomena are to be represented. Otherwise, assessing modelling choices regarding the external mode is mainly a matter of determining what numerical method is fastest for the space resolution considered.

As regards the filter of the checkerboard mode, the expression suggested by Killworth et al. (1991) is more appropriate than a classical Laplacian smoother, because it entails less perturbations of the impermeability conditions of the surface or the bottom of the ocean.

It was shown that the ocean surface elevation is mainly determined by a static equilibrium between two pressure forces. The forces resulting from the water motion, such as the Coriolis force, are much smaller, except in the Southern Ocean.

Finally, the first variational method, if interpreted as a way of evaluating the surface elevation from hydrographic data, was shown to be somewhat less accurate than the classical method. It has, however, some significant advantages. Indeed, it does not require any a priori knowledge of the depth of a hypothetical level of no geostrophic motion and it is capable of providing an estimate of the elevation over the entire ocean surface. It is thus far from clear that the classical approach should be preferred. The second variational method should be further investigated to see whether it could be used to evaluate the transport. If so, it might well outdo the two other approaches considered herein.

Acknowledgements. The development of ASTR's Ocean General Circulation Model (OGCM) is carried out within the scope of the impulse programme Global Change (Belgian State, Prime Minister's Services, Science Policy Office, Contract GC/10.013), the Convention d'Actions de Recherches Concertées No 92,97-154 with the Communauté Française de Belgique, the impulse programme Information Technology (Belgian State, Prime Minister's Services, Science Policy Office, Contract IT/SC/20), and a study contract between IBM of Belgium s.a. and the Université Catholique de T.ouvain (which allows us to use an IBM RS $6000-550$ workstation). Financial support for Jean-Michel Campin is provided by an EC grant. We had very interesting discussions with Pascale Delecluse, 
Peter Killworth, and Gurvan Madec about the development and the use of OGCMs. Many thanks are due to Thierry Fichefet and Stéphane Hovine for their constant help. We are indebted to Pierre Yves Le Traon for kindly providing us with Topex/Poseideon data

Topical Editor H. LeTreut thanks two referees for their help in evaluating this paper.

\section{Appendix A}

The time-slepping of our model is based on the split-explicit method. The latter consists in integrating the external and internal modes with different time steps, $\Delta t_{E}$ and $\Delta t_{I}$, each time increment being selected according to the fastest propagating phenomenon encountered in the mode considered. While the baroclinic mode is advanced by one time step, $N\left(=\Delta t_{2}, \Delta t_{E}\right)$ external time steps are performed. Typically, $10 \leqq N \leqslant 10^{2}$. In the simulations discussed here we had $N=30$. While performing the time-stepping of the external mode, the quantities related to the internal mode and those supposed to play a minor role are held fixed. In other words, within a given internal mode time increment, $\boldsymbol{F}_{p}$ and $\boldsymbol{A}$ are considered constant for the external time-marching. In principle, $D(\boldsymbol{U})$ should also be fixed while integrating the external mode for the $N$ external time steps lying within onhe internal increment. Like Killworth et al. (1991), we found that allowing $\boldsymbol{D}\left(\boldsymbol{U}^{\gamma}\right)$ to be evaluated at each external time step rendered the solution smoother and more stable. As a consequence, it is recommended to freeze $\boldsymbol{F}_{p}$ and $\boldsymbol{A}$ only, and to update $\boldsymbol{D}(\boldsymbol{U})$ at each external time increment.

The external mode equations Eqs. (7) and (9) are ordinary shallow water equations, with a minor difference in the nature of the forcing terms. Consequently, the numerical space-time discretization developed for those models may be utilized for solving Eqs. (7) and (9).

The equations of the internal and external modes are discretized on the B-grid according to the finite volume technique. The advection terms of scalar quantities are discretized so as to allow the future implementation of sophisticated schemes, with variable upwinding rate and flux limiters. The time-stepping involves only two time levels, and is thus not of leapfrog type. The external and internal gravity waves are discretized according to the forward-backward method (Mesinger and Arakawa, 1976; Beckers and Deleersnijder, 1993). The rertical fluxes may be evaluated implicitly, which allows using fine vertical resolution if needed (Delecluse et al., 1993).

When an efficient convergence accelerator was tried as suggested hy Bryan (1984), it was found necessary to apply a time filter to the batotropic variables before introducing them in the baroclinic mode equations. This filter consists of a time-averaging procedure that is somewhat similar to that advocated in Deelersnijder (1993), although the latter was suggested for different reasnns. We believe that the need for a time filter reflects an aliasing problem, arising because the external mode variables contain frequencies that cannot be resolved by the coarser time sampling of the internal mode. The filter enabled us to use a much larger internal time increment, whether or not in the framework of convergence acceleration. This topic will be addressed in detail in another article.

On the B-grid the solution is prone to numerical noise (Batteen and Han, 1981). Specifically, for the external mode, the B-grid may lead, under certain circumstances, to the splitting of the elevation "into two checkerboard subgrids with separate time development" (Killworth et al., 1991).

\section{References}

Batteen, M. L., and Y.-J. Han, On the computational noise of finite-difference schemes used in ocean models, Tellus, 33, 387-396, 1981.

Beckers, J.-M., Application of the GHER 3D general circulation model to the Western Mediterranean, J. Mar. Syst., 1, 315-332, 1991.
Beckers, J.-M. and E. Deeleersnijder, Stability of a FBTCS scheme applied to the propagation of shallow-water inertia-gravity waves on various space grids, $J$. Comput. Phys, 108, 95-104, 1993.

Berger, A., J.-M. Campin, E. Deleersnijder, M. EJ Mohajir, T. Fichefet, J.-F. Foccroulle, H. Grenier, M. Morales Maqueda, P. Tulkens, and .J.-P. van Ypersele, Modelling of the climatic system and its response to human activities, Proc Coll. "Global Change" (Vol. II), (Palais des Congrès, Bruxelles, 17-18 mai 1993), Beigian Science Policy Office, pp. 7-29, 1993.

Blumberg, A. F., and G. L. Mellor, A description of a three-dimensional coastal ocean circulation model, in Three-dimensinnal coastal ocean models, Ed. N. S. Heaps. American Geophysical Union, Washington, D.C, pp. 1-16, 1987.

Bryan, K., A numerical method for the study of the circulation of the World Ocean, J. Comput. Phys., 4, 347-376, 1969

Bryan, K., Accelerating the convergence to equilibrium of oceanclimate models, $J$. Phys. Oceanogr., 14, 666-673. 1984.

Cox, M., A primitive equation, three-dimensional model of the ocean, GFDL Ocean Group, Tech. Rep. No. 1, GFDL NOAA, Prínceton: 1984.

Delecluse, P., G. Madec, M. Imbard, and C. Levy, OPA Version 7 Ocean General Circulation Model: reference manual, LODYC. Rapport Interne 9305, Unirersité de Paris VI, Paris, 1993.

Deleersnijder, E., Numerical mass conservation in a free-surface sigma coordinate marine model with mode splitling, $J$. Mar. Syst., 4, 365-370, 1993.

Deleersnifjer, E., and J.-M. Campin, Du calcul de la position de la surface de l'océan dans un modèle de circulation générale, Contribution No. 70, Institut d'Astronomie et de Géophysique G. Lemaître, Université Catholique de Louvain, Louvain-la-Neuve, 1993.

Deleersnijder, E., J.-P. van Ypersele, and J. -M. Campin, An orthogonal curvilinear coordinate system for a World Ocean model, Ocean Modelling, 100, 7-10 (+ figures), 1993.

Dukowicz, J. K., R. D. Smith, and R. C. Malone, A reformulation and implementation of the Bryan-Cox-Semtner ocean model on the Connection Machine, J. Atmos. Ocean. Technol., 10, 195-208, 1993.

Gadd, A. J., A split-explicit integration scheme for numerica] weather prediclion, Q.J.R. Meteorol Soc., 104, 569-582, 1978.

Gill, A. E., Atmosphere-ocean dynamics, Academic Press, San Diego, 1982.

Gresho, P. M., and R. L. Sani, On pressure boundary conditions for the incompressible Navier-Stokes equations, Int. J. Numer. Methods Fluids. 7, 1111-1145, 1987.

Hellerman, S., and M. Rosenstein, Normal monthly wind stress over the world ocean with error estimates, J. Phys. Oceanogr., 13, 1093-1104, 1983.

Holland, W. R., and P. Malanotte-Rizzoli, Along-track assimilation of altimeter data into an ocean circulation model: space versus time resolution studies, J. Phys. Oceanogr., 19, 1507-1534, 1989.

Killworth, P. D., and J. M. Smith, Gradual instability of relaxationextrapolation schemes, Dyn. Atmos. Oreans, 8, 185-213, 1984.

Killworth, P. D., D. Stainforth, D. J. Webh, and S. M. Paterson, The development of a free-surface Bryan-Cox-Semtner ocean mdoel, J. Phys. Oceanogr. 21, 1333-1348, 1991.

Levitus, S., Climatological atlas of the world oceans, NOAA Prof Paper 13, U.S. Gov. Print. Office, Washington, D.C., 1982

Madala, R. V., Efficient time integration schemes for atmosphere and ocean models, in Finite-difference techniques for vectorized fuid dynamics calculations, Ed. D. T. Book, Springer, Berlin, Heidelberg, New York: pp. 56-74. 1981.

Mellor, G. L., and T. Ezer, A Gulf Stream model and an altimetry assimilation scheme, J. Geophys. Res., 96, 8779-8795, 1991.

Mesinger, F., and A. Arakawa, Numerical methods used in atmospheric models, GARP Publications Series (No. 17, Vol. 1), WMO-ICSU Joint Organizing Committee, 1976.

Pacanowski, R. C., and S. G. H. Philander, Parameterization of vertical mixing in numerical models of tropical oceans, J. Phys. Oceanogr., 11, 1443-1451, 1981. 
Peyret, R.. and T. D. Taylor, Computational methods for fluid flow, Springer Beriin, Heidelberg, New York, 1983.

Pinardi, N., A. Rosati, and R. C. Pacanowski, The sea surface pressure formulation of rigid lid models, Implications for altimetric data assimilation studies, J. Mar. Syst., 6, 109-119, 1995.

Ponte, R. M., Variability in a homogeneous global ocean forced by barometric pressure, Dyn. Atmos. Oceans, 18, 209-234, 1993.

Semtner, A. I., An oceanic general circulaion model with bottom topography, Tech. Rep. No. 9, Dept. of Meteorol., Univ, of Calif., Los Angeles, 1974.
Semtner, A. J., Finite-difference formulation of a World Ocean model, in Adnanced Physical Oceanographic Modelling, Ed. J. J. O'Brien, Reidel, Dordrecht, pp. 187-202, 1986

Schröter, J., U. Seiler, and M. Wenzel, Variational assimilation of Geosat data into an eddy-resolving model of the Gulf Stream extension area, J. Phys. Oceanogr., 23, 925-953, 1993.

Verron, J., Nudging satellite altimeter data into quasi-geostrophic ocean models, J. Geophys. Res., 97, 7479-7491, 1992.

Verron, J., J.-Ml. Molines, and E'. Blayo, Assimilation of Geosat data into a quasigenstrophic model of the North Atlantic between 20 and $50^{\circ} \mathrm{N}$ : preliminary results, Oceanol. Acta, 15, 575-583, 1992. 